\title{
РАЗРЫВ В ОЖИДАЕМОЙ ПРОДОЛЖИТЕЛЬНОСТИ ЖИЗНИ МУЖЧИН И ЖЕНЩИН: ОБЗОР ГЕНЕТИЧЕСКИХ, СОЦИАЛЬНЫХ И ЦЕННОСТНЫХ ФАКТОРОВ
}

\author{
ЮЛИЯ ЗИНЬКИНА, АНДРЕЙ КОРОТАЕВ
}

\begin{abstract}
Женское преимущество в ожидаемой продолжительности жизни (ОПЖ) встречается во всем мире, несмотря на различия в условиях жизни, положении женщин и действие других факторах. Однако это преимущество уменьшилось в последние годы в странах с низким уровнем смертности. При этом давно установлено, что, хотя женщины живут дольше мужчин, уровень заболеваемости (morbidity) у них выше. Сущуествует значительное число исследований, позволяющих с уверенностью утверждать, что в основе данного парадокса лежат как биологические факторы, так и соииальные явления и проиессы. Среди биологических факторов отмечаются гормональные (преобладание эстрогенов по сравнению с андрогенами, более низкий уровень у женщин тестостерона, связанного с рискованным поведением) и кариотипические различия (к примеру, прогрессивное искажение инактивации Х-хромосомы, истощение теломер, митохондриальное наследование и др.). Значительный вклад вносит поведение в области здоровья: мужчины реже обращаются к врачам, реже контролируют уровень холестерина и кровяное давление, имеют менее здоровый рацион и чаще склонны к рискованному поведению. Особый вклад вносят курение (в России, по некоторым оценкам, обусловливает до 5 лет разрыва в ОПЖ между мужчинами и женщинами, для Европь также отмечается как наиболее важная причина гендерных различий в смертности) и потребление алкоголя (мужчины пьют чаще, чем женщины, а пьющие мужчины употребляют алкоголь чаще, в больших количествах и с более высоким риском неблагоприятных последствий, чем пьюшие женщины; в ряде стран этот фактор объясняет до 30\% гендерного разрыва в ОПЖ). Ценностные объяснения факторов гендерных различий в ОПЖ не ограниченьл лишь данньли кросс-начиональных обследований ценностей. Представляется, что в эту же категорию целесообразно отнести объяснения, связанные с доминирующими представлениями о мужественности (маскулинности).
\end{abstract}

Ключевые слова: гендерный разрыв в ОПЖ, биологические факторы гендерного разрыва в ОПЖ, сочиильные факторы гендерного разрыва в ОПЖ, курение как фактор гендерного разрыва в ОПЖ, потребление алкоголя как фактор гендерного разрыва в ОПЖ.

Хотя размер гендерного разрыва и ожидаемая продолжительность жизни мужчин и женщин значительно различаются в разных странах мира, женщины живут дольше мужчин почти в каждой стране, в том числе в России (Андреев 2001; Хасанова 2019; Школьников, Милле, Вален 1995; Школьников, Андреев, Малева 2000: 20-23; Харченко, Михайлова, Онищенко 2003; Халтурина, Коротаев 2013; Школьников и др. 2014); в 1994 г. ожидаемая продолжительность жизни (ОПЖ) российских женщин превышала мужскую на 13 лет; предлагалось объяснение, что это различие обусловлено разной подверженностью полов макроэкономическому стрессу (Школьников, Андреев, Малева 2000: 21, 23).

ЮЛИЯ ВИКТОРОВНА ЗИНЬКИНА (juliazin@list.ru), РОССИЙСКАЯ АКАДЕМИЯ НАРОДНОГО ХОЗЯЙСТВА И ГОСУДАРСТВЕННОЙ СЛУЖБЫ ПРИ ПРЕЗИДЕНТЕ РОССИЙСКОЙ ФЕДЕРАЦИИ, РОССИЯ.

АНДРЕЙ ВИТАЛЬЕВИЧ КОРОТАЕВ (akorotaev@hse.ru), НАЦИОНАЛЬНЫЙ ИССЛЕДОВАТЕЛЬСКИЙ УНИВЕРСИТЕТ «ВЫСШАЯ ШКОЛА ЭКОНОМИКИ», РОССИЙСКАЯ АКАДЕМИЯ НАРОДНОГО ХОЗЯЙСТВА И ГОСУДАРСТВЕННОЙ СЛУЖБЫ ПРИ ПРЕЗИДЕНТЕ РОССИЙСКОЙ ФЕДЕРАЦИИ, РОССИЯ.

СТАТЬЯ ПОДГОТОВЛЕНА В РАМКАХ ВЫПОЛНЕНИЯ НАУЧНО-ИССЛЕДОВАТЕЛЬСКОЙ РАБОТЫ ГОСУДАРСТВЕННОГО ЗАДАНИЯ РАНХиГС.

СТАТЬЯ ПОСТУПИЛА В РЕДАКЦИЮ В ДЕКАБРЕ 2020 Г. 
Предыдущие исследования тенденций гендерного различия в смертности показали, что в течение первых трех четвертей XX века гендерный разрыв увеличился в большинстве промышленно развитых стран (Nathanson 1975; Waldron 1985; Rigby, Dorling 2007). К примеру, в 1955 г. Джефф Н. Моррис показал, что во второй половине XIX века в Соединенном Королевстве (Великобритания) показатели смертности были высокими и увеличивались среди людей среднего возраста (Morris 1955). У мужчин смертность была лишь на $10 \%$ выше, чем у женщин того же возраста. После этого благодаря улучшениям условий жизни уровень смертности начал падать с 1900 г. и продолжал снижаться до 1920-х годов. Начиная с 1920-х годов, показатели смертности все более расходились в зависимости от пола: показатели смертности среди мужчин не изменились, а среди женщин они продолжали снижаться. В 1950-х годах мужчины среднего возраста имели почти вдвое более высокий уровень смертности по сравнению с женщинами того же возраста. Моррис показал, что смертность от всех причин, кроме ишемической болезни сердца и рака дыхательной системы, среди мужчин продолжала снижаться с 1920 по 1950 г., что позволяет предположить, что увеличение различий в смертности мужчин и женщин населения Великобритании было связано прежде всего с началом современной эпидемии коронарной болезни сердца и рака легких, вызванной ростом курения среди мужчин (Morris 1955). В США разрыв в ожидаемой продолжительности жизни мужчин и женщин составлял 1 год в 1920 г. и вырос до 7,8 лет к 1975 г. (Arias 2007).

С 1970-х годов различия в ОПЖ мужчин и женщин начинают сокращаться в большинстве развитых стран, хотя время начала сужения этих различий в смертности менялось от страны к стране (Glei, Horiuchi 2007). Только в Японии разница в ожидаемой продолжительности жизни мужчин и женщин продолжает увеличиваться из-за относительно небольших улучшений смертности от болезней кровообращения и увеличения смертности от рака и внешних причин среди мужчин (Oksuzyan, BrønnumHansen, Jeune 2010). Что касается развивающихся стран, заслуживает внимания исследование М. Каллена и соавторов, разделивших 182 страны мира на 5 групп в зависимости от их положения в 2010 г. в рейтинге Индекса человеческого развития, модифицированного таким образом, чтобы исключить уровень ОПЖ в качестве основной меры, чтобы избежать автокорреляции (наиболее развитая группа 1). Переходя от развитых стран к странам с низким и средним уровнем дохода, Каллен с соавторами выделили три различных модели. В наиболее развитых из них (группа 2, включая такие страны, как Бразилия, Мексика, Таиланд и ЮАР) соотношение мужской и женской ОПЖ демонстрировало устойчивый спад в течение 1970-2010 гг., напоминающий страны группы 1 в период с 1900 по 1970 г. Далее в странах группы 2 последовал предполагаемый разворот этого тренда в 2000 г., похожий на смену тренда в странах группы 1 примерно 2-3 десятилетия назад. Страны группы 3, напротив, демонстрируют высокое соотношение мужской и женской ОПЖ вплоть до снижения, которое, по-видимому, начинается примерно в 1990-2000 гг.; в странах же группы 4 (самых бедных в мире) соотношение мужской и женской ОПЖ остается высоким в течение всего периода (страны Африки к югу от Сахары) (Cullen et al. 2015).

Женское преимущество в ОПЖ встречается во всем мире, несмотря на различия в условиях жизни, положении женщин и других факторах. Однако это преимущество 
уменьшилось в последние годы в странах с низким уровнем смертности. Лишь немногие исследователи смотрели на гендерный разрыв в ОПЖ в пожилом возрасте (65 лет) в более длительной исторической перспективе. Одной из таких работ является исследование М. Торслунда и соавторов, в котором были оценены данные по ОПЖ для 17 стран из базы данных Human Mortality Database с 1751 по 2007 г. Поскольку большинство изменений в ОПЖ, происходящих сегодня, обусловлено снижением смертности в старости, гендерное различие в ОПЖ было рассчитано в возрасте 65 лет. Большинство стран с низкой смертностью демонстрируют одинаковую историческую тенденцию: рост и последующее падение преимуществ женщин в ОПЖ в возрасте 65 лет. Исследователями были выделены три этапа, которые прошли все рассмотренные страны, кроме двух (Японии и Испании). После долгой фазы, на протяжении которой женщины имели преимущество в ОПЖ в 65 лет размером менее 1 года, гендерный разрыв значительно увеличился в течение XX века. Увеличение произошло во всех странах, но в разные моменты времени. В некоторых странах, таких как Англия и Франция, преимущество женщин начало расти раньше (19001919 гг.); 50 лет спустя это произошло в Швеции, Норвегии и Нидерландах. Наконец, на третьей фазе последовало более синхронное падение женского преимущества в исследованных странах к концу столетия, за исключением Японии и Испании (Thorslund et al. 2013).

При этом давно установлено, что, хотя женщины живут дольше мужчин, уровень заболеваемости (morbidity) у них выше (Браун, Панова, Русинова 2007; Андреев, Школьников, МакКи 2002). Мужчины испытывают больше опасных для жизни хронических заболеваний и умирают моложе, тогда как женщины живут дольше, но имеют больше несмертельных острых и хронических состояний и инвалидности, оказывающих заметное отрицательное влияние на качество их жизни в старших возрастах. Так, статистические данные по США в начале 2000-х годов показывали, что три основные причины смерти с поправкой на возраст одинаковы для мужчин и женщин (болезни сердца, рак и инсульт). Однако у мужчин наблюдались более опасные для жизни хронические заболевания в более молодом возрасте, включая ишемическую болезнь сердца, рак, цереброваскулярные заболевания, эмфизему, цирроз печени, заболевание почек и атеросклероз. Напротив, женщины сталкивались с более высоким уровнем хронических расстройств, таких как аутоиммунные заболевания и ревматологические расстройства, а также с менее опасными для жизни заболеваниями (National Center... 2003).

Существует значительное количество исследований, позволяющих в совокупности с уверенностью утверждать, что в основе данного парадокса лежат как биологические факторы, так и социальные явления и процессы. Однако на настоящий момент в нашем понимании отсутствует достаточно полное и системное объяснение того, как сочетаются и взаимодействуют друг с другом социальные и биологические факторы, создавая эти парадоксальные различия в здоровье и продолжительности жизни мужчин и женщин. 


\section{ГЕНДЕРНЫЙ РАЗРЫВ В ОЖИДАЕМОЙ ПРОДОЛЖИТЕЛЬНОСТИ ЖИЗНИ: БИОЛОГИЧЕСКИЕ ФАКТОРЫ И ОБЪЯСНЕНИЯ}

Предполагается, что различия в биохимических конституциях, такие как более высокий уровень эстрогена у женщин, способствуют гендерному дисбалансу. Эстроген защищает женщин от сердечно-сосудистых заболеваний до наступления менопаузы (Philips 2006). Было показано, что биологические факторы влияют на гендерный разрыв в ОПЖ, особенно в младенческом возрасте (Kaplan, Erickson 2000).

Недавние публикации связывают рискованное поведение с уровнями тестостерона. Было показано, что тестостерон оказывает влияние на широкий спектр социальных форм поведения, включая доминирование и агрессию (Mehta, Josephs 2011), и модулирующее влияние на эмоциональные и поведенческие реакции на угрозу (van Wingen et al. 2011). Более высокий уровень тестостерона коррелирует с более низкими оценками серьезности и распространенности угроз для здоровья (Ristvedt, Josephs, Liening 2012), а также связан со стремлением к вознаграждению и игнорированием угрозы (Stanton, Liening, Schultheiss 2011).

Было обнаружено, что аспекты процесса старения у мужских и женских особей одного и того же вида млекопитающих различны. Тем не менее лежащие в основе механизмы этих половых различий в здоровье и продолжительности жизни остаются недостаточно понятными, и неясно, какие аспекты этого диморфизма обусловлены гормональными различиями (т. е. преобладанием эстрогенов по сравнению с андрогенами) или кариотипическими различиями (т. е. набором хромосом XX по сравнению с XY) (Sampathkumar et al. 2020).

Женщины имеют генетическое преимущество при зачатии: хотя половой диморфизм в долголетии наблюдается не у всех видов животных, он имеет тенденцию отдавать предпочтение гомогаметному полу (например, у людей - женщинам, у птиц - мужским особям) по сравнению с гетерогаметным полом. Инактивация Х-хромосомы обычно защищает от двойной дозы экспрессии гена X-хромосомы у женщин; однако это также защищает женщин от неблагоприятных генов на первой X-xромосоме (Marais et al. 2018).

Истощение теломер (т. е. прогрессирующее уменьшение длины хромосом, которое происходит с клеточным делением / репликацией и повреждением) коррелирует с более короткой продолжительностью жизни некоторых видов животных. У людей у взрослых мужчин теломеры короче, чем у женщин (Gardner et al. 2014).

Митохондрии люди наследуют только от матерей; было высказано предположение, что митохондриальный геном оптимизировался для функционирования с женским геномом посредством естественного отбора, действующего преимущественно на взаимодействия митохондриального и нуклеарного генома у женщин. Такая оптимизация митохондриальной «приспособленности» в женских клетках может обеспечить преимущество в продолжительности жизни, учитывая, что митохондриальная дисфункция вовлечена в старение и заболевания, такие как рак, сердечно-сосудистые и нейродегенеративные заболевания. 
Половые гормоны: после зачатия и генетического определения пола основными модуляторами полового развития являются эндогенные половые гормоны - тестостерон и эстроген. Гормональные влияния на женскую биологию в период раннего развития плода и в детстве, а также после полового созревания приводят к благоприятным для женщин отличиям от мужчин в иммунной функции, окислительном стрессе и антиоксидантном статусе, метаболизме липопротеинов, накоплении и метаболизме липидов, гипоталамогипофизарно-надпочечниковом (HРА) ответе на стресс, а также способности женских клеток поддерживать целостность перед лицом нескольких стрессов. Комбинация этих факторов может быть компонентом преимущества ОПЖ женщин (Seifarth, McGowan, Milne 2012; Austad 2006).

\section{ГЕНДЕРНЫЙ РАЗРЫВ В ОЖИДАЕМОЙ ПРОДОЛЖИТЕЛЬНОСТИ ЖИЗНИ: ПОВЕДЕНЧЕСКИЕ ФАКТОРЫ И ОБЪЯСНЕНИЯ}

Многие ученые-медики утверждают, что поведение в отношении здоровья является одним из наиболее важных факторов, влияющих на здоровье, и что изменение поведения в отношении здоровья является «наиболее эффективным способом» предотвращения болезней (Коор 1996).

Использование возможностей системы здравоохранения: регулярные медицинские осмотры имеют решающее значение для раннего выявления многих потенциально смертельных заболеваний. Мужчины посещают врачей реже, чем женщины, и пользуются значительно меньшим количеством медицинских услуг.

Профилактика заболеваний: мужчины реже, чем женщины, проверяют уровень холестерина и свое кровяное давление. Поскольку мужчины с меньшей вероятностью проходят скрининг или регулярно посещают врача, на 20\% меньше мужчин, чем женщин, получают лечение от гипертонии, и женщины в 1,5 раза чаще, чем мужчины, держат гипертонию под контролем (Courtenay 2000).

Другие формы профилактического ухода: меньше мужчин, чем женщин с гипертонией, пытаются контролировать ее, ограничивая потребление соли, уменьшая вес или занимаясь физическими упражнениями. Мужчины реже, чем женщины, принимают витаминные добавки, хотя у них зачастую менее питательный рацион, чем у женщин. Мужчины потребляют значительно меньше клетчатки, фруктов и овощей, чем женщины.

Жиры и холестерин: ограничение жиров в рационе считается основным средством для улучшения здоровья, снижения веса и предотвращения болезней сердца, инсульта, диабета и рака. Мужчины всех возрастов потребляют больше насыщенного жира и пищевого холестерина, чем женщины, даже если половые различия скорректированы с учетом размера тела.

Рискованное поведение: мужчинам чаще присущи опасное вождение, вождение в нетрезвом виде, отказ от использования ремней безопасности (как при вождении, так и при поездках в качестве пассажира), отказ от использования шлема при поездках на 
велосипеде, драки, владение оружием и его использование, криминальная деятельность и др. (Courtenay 2000).

\section{ГЕНДЕРНЫЙ РАЗРЫВ В ОЖИДАЕМОЙ ПРОДОЛЖИТЕЛЬНОСТИ ЖИЗНИ: КУРЕНИЕ КАК ПОВЕДЕНЧЕСКИЙ ФАКТОР}

Исследование Дж. МакКартни и его соавторами величины гендерного разрыва в смертности от всех причин в 30 европейских странах и вклада смертей, связанных с курением и алкоголем, показало, что в Европе наблюдался значительный разброс величины «избытка» мужской смертности от всех причин на 100 тыс. человек в год - от 188 в Исландии до 942 на Украине. Смертность, связанная с курением, обусловливала от 40 до $60 \%$ гендерного разрыва. Курение оставалось наиболее важной причиной гендерных различий в смертности в Европе. Изменения в структуре курения по полу предполагают, что гендерный разрыв в смертности уменьшится в ближайшие десятилетия (McCartney et al. 2011).

О роли курения в разрыве ОПЖ мужчин и женщин исследователи говорят уже достаточно долго. Так, Т. Вальконен и Ф. ван Поппель в своем исследовании пяти стран Северной Европы пришли к следующим оценкам: вклад курения в межполовые различия в ОПЖ в возрасте 35 лет в период 1970-1989 гг. варьировался от 14\% в Швеции в 19851989 гг. до 72\% в Нидерландах в 1970-1974 гг. (Valkonen, van Poppel 1997). Оценки для населения США, на которые ссылаются большинство существующих исследований, варьируются от 50 до 62\% для 1960-х и 1970-х годов (Retherford 1972; Waldron 1986) и от 22 до 25\% для 1990-х (Rogers et al. 2010).

Исследование населения 44 европейских стран за 55-летний период наблюдений (с 1955 по 2009 г.) позволило оценить влияние отдельных компонентов, в том числе курения и других небиологических факторов, на тенденции и общие масштабы гендерного разрыва в ОПЖ. Разница в ОПЖ по полу варьировалась от 1,7 года в Македонии в 1955-1959 гг. до 13,2 года в России в 2000-2004 гг. Оценки воздействия биологических факторов варьируются от 0,5 до 1,6 года. Соответственно, большая часть разницы в ожидаемой продолжительности жизни по полу должна быть отнесена на счет небиологических факторов, одним из которых является курение. Наименьшее оценочное влияние курения на межполовые различия в ОПЖ составляет 0,02 года для Таджикистана в 1955-1959 гг., а самое высокое - 5,0 лет для России в 1990-1994 гг. (Luy, Wegner-Siegmundt 2013). Другое исследование подтверждает, что в России курение обусловливает более 5 лет разрыва в ОПЖ мужчин и женщин, при этом 2,5-3 года разрыва в ожидаемой продолжительности здоровой жизни (ОПЗЖ) (Кузнецова 2019). В Белоруссии в среднем за 1950-2009 гг. женщины переживали мужчин на 10,1 года. Вклад курения составил 3,9 года, а других небиологических факторов - 3,8 года (Luy, Wegner-Siegmundt 2013).

Более позднее исследование 31 европейской страны дало более подробные результаты. В 2014 г. межполовая разница в ОПЖ при рождении составляла в среднем 7,0 лет в 31 изученной европейской стране, но значительно варьировалась (от 11,2 года в России до 3,0 лет в Исландии). Смертность, связанная с курением, в среднем обусловливала 
3,0 года $(43,5 \%)$ в отношении межполовой разницы в ОПЖ при рождении. Абсолютный вклад смертности от курения был самым высоким в России (5,3 года) и самым низким в Швеции (ноль) и Исландии $(-0,1)$. Относительный вклад смертности от курения составлял $50 \%$ или выше в Греции, Болгарии, Хорватии, Венгрии и на Украине. В среднем вклад смертности, связанной с курением, в межполовую разницу в ОПЖ при рождении составил 4,6 года $(47,5 \%)$ в Восточной Европе, 2,0 года (39\%) в Южной Европе, 1,3 года (28\%) в Западной Европе и 0,4 года (10\%) в Северной Европе (Janssen 2020).

При этом ни межполовая разница в ОПЖ при рождении, ни вклад смертности от курения не были стабильными с течением времени. В 1956 г. межполовая разница в ОПЖ при рождении была меньше, чем в 2014 г., в большинстве стран; в то время как вклад смертности от курения был больше, за исключением Франции, Португалии, Испании и Венгрии. Средняя межполовая разница в ОПЖ при рождении составляла 7,8 года в 1985 г. и была самой высокой в 1995 г. (9,0 года). Средний относительный вклад связанной с курением смертности в половые различия в ОПЖ в 1985 г. составил почти 60\%, после чего снизился. Абсолютный вклад смертности, связанной с курением, был в среднем самым высоким примерно в 1995 г. (5,2 года), после чего снижался параллельно с тенденцией к снижению межполовой разницы в ОПЖ при рождении (Janssen 2020).

\section{ГЕНДЕРНЫЙ РАЗРЫВ В ОЖИДАЕМОЙ ПРОДОЛЖИТЕЛЬНОСТИ ЖИЗНИ: УПОТРЕБЛЕНИЕ АЛКОГОЛЯ КАК ПОВЕДЕНЧЕСКИЙ ФАКТОР}

В каждом обществе, где употребление алкоголя женщинами и мужчинами измерялось или обследовалось, мужчины пьют больше, чем женщины, а пьянство у мужчин приводит к большему количеству социальных проблем, чем у женщин. В большинстве исследований, посвященных употреблению алкоголя мужчинами и женщинами, изучались общества в Северной Атлантике, однако сообщения о том, что употребление алкоголя в больших количествах и связанные с этим проблемы являются преимущественно моделями поведения мужчин, также поступили из разных регионов Африки, Азии, Океании и Латинской Америки (Wilsnack et al. 2000).

В большинстве стран Европы мужчины сообщали, что употребляли в 2-4 раза больше алкоголя, чем женщины. Соотношения полов для различных типов напитков варьировались, как и частота употребления алкоголя. Мужчины также сообщали о сильном эпизодическом употреблении алкоголя примерно в 3-6 раз чаще, чем женщины. В Северной Европе это соотношение было несколько меньше, чем в других странах, т. е. разница между мужчинами и женщинами в употреблении большого количества алкоголя была меньше. Это имело место во всех возрастных группах. Во всей Европе наименьшее соотношение полов было обнаружено в молодых возрастных группах, т. е. в более старших возрастных группах гендерный разрыв в употреблении алкоголя в больших количествах был даже более выраженным, чем в младших возрастных группах. Таким образом, наименьшая разница между тяжелым эпизодическим алкоголизмом среди мужчин и женщин была обнаружена среди молодежи в северных странах (Mäkelä et al. 2006). 
Наборы данных Международной исследовательской группы по гендеру и алкоголю (IRGGA) подтверждают некоторые основные результаты более ранних исследований, сравнивающих поведение мужчин и женщин с алкоголем. Исследование Р. Уилснека и соавторов на этих данных показало, что мужчины пили чаще, чем женщины, а пьющие мужчины употребляли алкоголь чаще, в больших количествах и с более высоким риском неблагоприятных последствий, чем пьющие женщины. Постоянные доказательства этих гендерных различий в обществах, где исторические и культурные изменения сделали алкоголь более доступным для женщин (например, благодаря более высокой экономической активности женщин), указывают на то, что любое сближение употребления алкоголя мужчинами и женщинами имело незначительное влияние на модели потребления алкоголя во многих обществах. Сохранение гендерных различий и постоянные закономерности таких различий на всех этапах взрослой жизни дают основание полагать, что гендерные различия в употреблении алкоголя, по крайней мере, частично связаны с биологическими различиями между женщинами и мужчинами (Wilsnack et al. 2000).

Более позднее исследование Р. Уилснека и соавторов ставило своей целью оценить многонациональные модели потребления алкоголя в зависимости от пола и возраста на данных крупных опросов населения в отношении употребления алкоголя у мужчин и женщин (n > 900) в 35 странах в 1997-2007 гг. Пьянство как таковое и употребление алкоголя в больших количествах были неизменно более распространены среди мужчин, чем среди женщин, а воздержание от алкоголя в течение жизни было неизменно более распространенным среди женщин. Среди респондентов, которые когда-либо были пьющими, женщины во всех возрастных группах неизменно чаще бросали пить, чем мужчины. Среди пьющих распространенность высокочастотного употребления алкоголя была неизменно наибольшей в самой старшей возрастной группе, особенно среди мужчин. Как и ожидалось, мужчины по-прежнему превосходят женщин в пьянстве и употреблении алкоголя в больших объемах, хотя соотношение полов варьируется (Wilsnack et al. 2009).

Исследование Дж. МакКартни и соавторов ставило перед собой цель сравнить величину гендерного разрыва в смертности от всех причин в 30 европейских странах и оценить вклад смертей, связанных с курением и алкоголем. Результаты показали, что в Европе наблюдался значительный разброс величины «избытка» мужской смертности от всех причин на 100 тыс. человек в год: от 188 в Исландии до 942 на Украине. Смертность, связанная с алкоголем, обычно обусловливала от 20 до 30\% гендерного разрыва в Восточной Европе и от 10 до 20\% в других странах Европы (McCartney et al. 2011; о потреблении крепких алкогольных напитков как наиболее сильном факторе избыточной смертности среди мужчин трудоспособного возраста в Европе см. также Korotayev et al. 2018).

Гендерные различия в ОПЖ традиционно были значительными в Центральной и Восточной Европе. Сравнительно недавно исследователи изучили роль алкоголя в гендерных различиях в ОПЖ в восьми странах: Эстонии, Литве, Латвии, Молдавии, Польше, Румынии, России и на Украине, измерив эту роль в период с 1965 по 2012 г. Результаты показали, что в этих странах преимущество женщин в ОПЖ по сравнению с мужчинами увеличилось в среднем с 7,3 года в 1965 г. до 10,0 года в 2012 г. Вся связанная с алкоголем смертность обусловила в среднем 1,9 года (интервалы неопределенности: 1,2- 
2,5; 18,8\%) гендерных различий в ОПЖ в период с 1990 по 2012 г. Ее относительный вклад в большинстве стран увеличивался примерно до 2005 г., а затем уменьшался, что привело к вкладу не менее 15\% в 2012 г. Абсолютный вклад алкоголя в гендерный фактор разрыва сильно коррелировал с общими гендерными различиями в ОПЖ, за исключением Польши и Эстонии (Trias-Llimós, Janssen 2018).

Исследования Т.В. Коссовой, Е.В. Коссовой и М.А. Шелунцовой (2018) показали, что сокращение разрыва в ОПЖ между мужчинами и женщинами в российских регионах не может быть достигнуто без сокращения объемов потребления алкоголя (см. также: Халтурина, Коротаев 2013).

Факторы, объясняющие различия в употреблении алкоголя мужчинами и женщинами, привлекали внимание многих исследователей, предлагавших разного рода гипотезы.

Что касается биологически обусловленных различий, имеется значительное число исследований, показывающих разницу в воздействии алкоголя на мужчин и женщин, связанную с гормонами, нейротрансмиттерами и др. Так, обзорное исследование 266 научных статей из баз MEDLINE, PubMed, Web od Science, SCOPUS, PsycINFO, CIHAHL, проведенное A. Эролом и В. Карпяком, подтверждает наличие различий, связанных с употреблением алкоголя и его влиянием на мужчин и женщин. У регулярно выпивающих женщин связанные с алкоголем проблемы развиваются реже, чем у регулярно выпивающих мужчин. Однако сильно пьющие женщины имеют больше проблем со здоровьем и психических расстройств, связанных с алкоголем, чем мужчины-пьяницы. По мере того, как новые поколения становятся все более гомогенизированными в отношении социальных, экономических и культурных характеристик, гендерный разрыв в паттернах употребления алкоголя становится менее значительным (Erol, Karpyak 2015).

Особый интерес представляет влияние алкоголя на уровень гормонов. Половые гормоны влияют на все системы организма прямо или косвенно через влияние на гипоталамо-гипофизарно-надпочечниковую ось, внося свой вклад в различия в поведении, в том числе связанные с алкоголем. Имеющиеся данные подтверждают прямое влияние острого употребления алкоголя на уровень тестостерона у мужчин, что может потенциально способствовать усилению агрессивности (которая, как известно, коррелирует с уровнем тестостерона) у мужчин во время алкогольной интоксикации (Erol, Karpyak 2015).

Гендерные различия в употреблении алкоголя слишком последовательны в направлении для того, чтобы объясняться исключительно социальными или культурными переменными, но слишком различаются по размеру для объяснений, основанных исключительно на биологических эффектах. Синтетическая точка зрения, которая признает как биологическое, так и культурное влияние на употребление алкоголя мужчинами и женщинами, может приблизиться к объяснению того, почему гендерные различия в употреблении алкоголя столь универсальны и так разнообразны. В качестве стимула для дальнейших исследований Р. Уилснек с соавторами предлагают возможный путь к такому теоретическому синтезу. Этот подход начинается с предположения, что общества 
используют биологические явления в качестве основы для создания правил социального поведения и отношений. Люди интерпретируют, систематизируют и уточняют то, что они наблюдают в природе, чтобы трансформировать различия в систематические и абсолютные классификации людей и поведения. Таким образом, общества неоднократно дихотомизируют поведение мужчин и женщин на основе предполагаемых внутренне присущих или биологических различий между полами. Уникальные способности женщин рожать и воспитывать детей обычно становятся основой для гораздо более сложных различий в социальных ролях мужчин и женщин. Даже небольшие наблюдаемые биологические различия в том, как мужчины и женщины реагируют на потребление алкоголя, могут обеспечить универсальную основу для культурных разработок, в которых указывается, что мужчины и женщины должны различаться в том, как они употребляют алкоголь и как они ведут себя, когда пьют. Небольшие гендерные различия в чувствительности к алкоголю могут быть использованы для обоснования не только более низких нормативных ограничений на количество, которое женщины должны потреблять, но также и более строгих ограничений на то, где и когда женщины могут пить. Такая культурная проработка склонна усиливать гендерные различия в поведении, связанном с употреблением алкоголя, но в разной степени и по-разному (Wilsnack et al. 2000).

Этнографические исследования показывают, что в тех случаях, когда гендерные роли наиболее четко разделены (например, где женщины в основном сидят дома, выполняя домашние обязанности), оказываются наиболее четко разделенными и паттерны женского и мужского потребления алкоголя. Мужчины могут пить больше, чем женщины, отчасти потому, что употребление алкоголя является демонстрацией мужественности: своим поведением после употребления алкоголя, мужчины могут (или могут быть обязаны) демонстрировать свою выносливость, самоконтроль, готовность рисковать. Употребление алкоголя группами, состоящими только из мужчин, рассматривается в таких случаях как важный способ, благодаря которому мужчины могут избежать контроля со стороны других, игнорировать социальные различия, получать социальную поддержку и устанавливать прочные личные связи друг с другом. На употребление алкоголя женщинами часто накладываются более жесткие социальные ограничения из-за страха, что пьянство может более негативно повлиять на социальное поведение и обязанности женщин, а также снизить социальный контроль над сексуальностью женщин. Опьяняющее воздействие алкоголя считалось несовместимым с традиционными домашними обязанностями женщин и могло указывать на опасный отказ от социального контроля над семейными отношениями и общественным поведением женщин (Wilsnack et al. 2000; Holmila, Raitasalo 2005).

\section{ГЕНДЕРНЫЙ РАЗРЫВ В ОПЖ И ЦЕННОСТИ}

Пол является ключевым компонентом в формировании жизни мужчин, и доминирующие идеи мужественности влияют на них через негативное поведение в отношении здоровья. Доминирующие представления о мужественности должны быть достигнуты и реализованы посредством взаимодействия в определенных социальных условиях. Большинство мужчин не соответствуют идеалам гегемонистской маскулинности, но мотивированы поддерживать 
эти идеалы или стремиться к их достижению. Таким образом, этот культурный идеал или выражение доминирующей мужественности легитимизируется и санкционирует существующую гендерную иерархию в целом (Hinote, Webber 2012).

Например, для приобретения власти от мужчин требуется, чтобы они подавляли свои потребности и отказывались признавать свою боль. Другие убеждения и виды поведения, связанные со здоровьем, которые могут быть использованы при демонстрации гегемонистской маскулинности, включают отрицание слабости или уязвимости, эмоциональный и физический контроль, проявление силы и крепости, отказ от любой потребности в помощи, постоянный интерес к сексу, проявление агрессивного поведения и физического доминирования. Эти связанные со здоровьем демонстрации пола и власти представляют собой формы практики власти на микроуровне (Courtenay 2000; Kaufman 1994; Pyke 1996). Показывая или воплощая гегемонистские идеалы маскулинности в поведении, связанном со здоровьем, мужчины укрепляют твердо устоявшиеся культурные убеждения, что мужчины более могущественны и менее уязвимы, чем женщины, и что самые влиятельные люди среди мужчин - это те, для кого здоровье и безопасность не имеют значения. Растущий объем исследований свидетельствует, что мужчины, которые поддерживают доминирующие нормы маскулинности, ведут себя хуже в отношении своего здоровья и имеют более высокий риск для здоровья, чем их сверстники с менее традиционными убеждениями (Courtenay 2000).

Один из способов, с помощью которого мужчины могут демонстрировать культурно-значимые (или доминирующие в данной культуре) формы мужественности - это отрицание уязвимости, принятие рисков, которые могут нанести вред их здоровью, и отказ от тех поведенческих паттернов и убеждений в области здоровья, которое в данной культуре ассоциируются с женщинами (Emslie, Hunt 2008). Так, мужчины в Соединенных Штатах страдают более тяжелыми формами хронических заболеваний, имеют более высокий уровень смертности по всем 15 основным причинам смерти и умирают почти на 7 лет моложе женщин. Связанные со здоровьем убеждения и поведение являются важными факторами, способствующими этим различиям. Мужчины в Соединенных Штатах с большей вероятностью, чем женщины, принимают убеждения и поведение, повышающие их риск, и менее склонны к поведению, связанному со здоровьем и долголетием. В попытке объяснить эти различия исследователи предлагают реляционную теорию здоровья мужчин с точки зрения социального конструкционизма. В рамках этой теории предполагается, что связанные со здоровьем убеждения и поведение, как и другие социальные практики, в которые вовлечены женщины и мужчины, являются средством для демонстрации женственности и мужественности. Эта теория предполагает, что поведение в отношении здоровья используется в повседневных взаимодействиях в социальной структуризации пола и власти. Далее предполагается, что социальные практики, которые подрывают здоровье мужчин, часто являются признаками мужественности и инструментами, которые мужчины используют при переговорах о социальной власти и статусе (Courtenay 2000).

В частности, модели и практика употребления алкоголя служат для поддержания и воспроизводства социальных структур, которые его закрепляют. Выходя за локальные контексты социального взаимодействия, парадигма, связанная с гегемонистской 
мужественностью, действует институционально через семью, рабочую среду и экономику. Силы социализации в семье рабочего класса были тесно связаны с развитием мужской идентичности, многое в которой было связано с алкоголем. Мальчиков поощряли или подталкивали к употреблению алкоголя в качестве ритуала перехода в мужчины, служащего для того, чтобы дистанцировать юношей от детей и от вмешивающегося материнского (т. е. женского) влияния. Неспособность применить эти ритуализированные методы вызывала социальные санкции, насмешки и разочарование. Через эти ранние проблемы мужественности отцы и более широкая сеть мужчин, окружающих семью, настоятельно поощряли молодых мужчин впитывать и обычно инициировали эти практики в возрасте от 15 до 19 лет. Неслучайно эти годы пересекались с началом трудовой жизни и вступлением молодого человека в ряды рабочей силы (Hinote, Webber 2012).

Подобно нездоровому поведению, доминирующие или идеализированные представления о маскулинности также предоставляют средства для демонстрации гендера. Эти признаки «настоящей» мужественности легкодоступны мужчинам, которые в противном случае могли бы иметь ограниченные социальные ресурсы для конструирования своей мужественности. Многими исследованиями было показано, что среди молодых людей в США такие факторы, как низкий уровень образования, низкий доход семьи и этническая принадлежность к афроамериканцам, связаны с традиционными доминирующими нормами маскулинности (Courtenay 2000).

Еще одно объяснение основано на концепции ограниченного выбора, отличающей социологию выбора от экономических моделей теории рационального выбора. Вкратце суть концепции такова: ограничения, влияющие на повседневный выбор и возможности сделать здоровье своим приоритетом, различны для мужчин и женщин. Соответственно, для объяснения гендерных различий в здоровье и ОПЖ необходимо изменить модели детерминант здоровья так, чтобы они учитывали эти ограничения. Так обеспечивается учет преобладающего в общественном здравоохранении понимания неравенства в отношении здоровья, а также роли личного выбора и поведения в отношении здоровья в усилении или уменьшении способности человека прожить долгую и здоровую жизнь. Ограниченный выбор может также повлиять на позитивное и негативное поведение в отношении здоровья и способов преодоления проблем, которые влияют как на психологическое, так и на физическое функционирование. Таким образом, гендерные различия в ограничениях способствуют неравенству в отношении здоровья как прямо, так и косвенно, влияя на выбор как мужчин, так и женщин и их совокупный биологический риск (Rieker, Bird 2005).

В целом можно отметить, что, хотя в последние десятилетия набор научных работ, изучающих гендерный разрыв в ОПЖ и его факторы, предлагающих возможные объяснения этого явления с позиции различных дисциплин, существенно расширился, остается верным наблюдение, сделанное Л. Фербрюгге и Д. Уингардом еще в 1987 г.: ни одна строго биомедицинская интерпретация данных о различии ожидаемой продолжительности жизни у мужчин и женщин, равно как и ни одна гипотеза, основанная исключительно на социальных факторах, не способны полностью объяснить данное явление (Verbrugge, Wingard 1987). Природа гендерного разрыва ОПЖ объясняется комплексом разноплановых факторов. 


\section{ЛИТЕРАТУРА}

Андреев Е.М. (2001). Смертность мужчин в России. Вопросы статистики, 7, 27-33.

Андреев Е.М., Школьников В.М., МакКи М. (2002). Продолжительность здоровой жизни. Bопросы статистики, 11, 16-21.

Браун Дж.В., Панова Л.В., Русинова Н.Л. (2007). Гендерные различия в здоровье. Социологические исследования, 6, 114-122.

Коссова Т.В., Коссова Е.В., Шелунцова М.А. (2018). Анализ факторов, определяющих различие в ожидаемой продолжительности мужчин и женщин в регионах России. ЭКО, 4, 116-132. DOI: http://dx.doi.org/10.30680/ECO0131-7652-2018-4-116-132

Кузнецова П.О. (2019). Курение как фактор сокращения ожидаемой продолжительности жизни в России. Демографическое обозрение, 6(3), 31-57. DOI: https://doi.org/10.17323/demreview.v6i3.9854

Халтурина Д. А., Коротаев А. В. (2013). Русский крест. Российский демографический кризис: факторы, механизмы, пути преодоления. М.: Либроком/URSS.

Харченко В.И., Михайлова Р.Ю., Онищенко П.И. (2003). Показатели продолжительности жизни населения России в сравнении с другими странами. Проблемы прогнозирования, $6,119-127$.

Хасанова Р.Р. (2019). Продолжительность жизни: дифференциация по полу. Экономическое развитие России, 26(5), 56-60.

Школьников В.М., Андреев Е.М., Макки М., Леон Д.А. (2014). Рост продолжительности жизни в России 2000-х годов. Демографическое обозрение, 1(2), 5-37. DOI: https://doi.org/10.17323/demreview.v1i2.1815

Школьников В., Андреев Е., Малева Т. (Ред.) (2000). Неравенство и смертность в России. М.: Сигналъ.

Школьников В., Милле Ф., Вален Ж. (1995). Ожидаемая продолжительность жизни и смертность населения России в 1970 - 1993 годах: анализ и прогноз. М.: Фонд «Здоровье и окружающая среда».

Arias E. (2007). United States Life Tables, 2004. National Vital Statistics Reports, 54(14), 1-40.

Cullen M.R., Baiocchi M., Eggleston K., Loftus P., Fuchs V. (2015). The weaker sex? Vulnerable men, resilient women, and variations in sex differences in mortality since 1900 (No. w21114). National Bureau of Economic Research.

Austad S.N. (2006). Why women live longer than men: sex differences in longevity. Gender medicine, 3(2), 79-92. DOI: https://doi.org/10.1016/s1550-8579(06)80198-1

Courtenay W.H. (2000). Behavioral factors associated with disease, injury, and death among men: Evidence and implications for prevention. The Journal of Men's Studies, 9(1), 81-142. DOI: https://doi.org/10.3149/jms.0901.81

Emslie C., Hunt K. (2008). The weaker sex? Exploring lay understandings of gender differences in life expectancy: a qualitative study. Social Science \& Medicine, 67(5), 808-816. DOI: https://doi.org/10.1016/j.socscimed.2008.05.009.

Erol A., Karpyak V.M. (2015). Sex and gender-related differences in alcohol use and its consequences: Contemporary knowledge and future research considerations. Drug and alcohol dependence, 156, 1-13. DOI: https://doi.org/10.1016/j.drugalcdep.2015.08.023 
Gardner M., Bann D., Wiley L., Cooper R., Hardy R., Nitsch D., Bekaert S. (2014). Gender and telomere length: systematic review and meta-analysis. Experimental gerontology, 51, 15-27. DOI: https://doi.org/10.1016/j.exger.2013.12.004

Glei D.A., Horiuchi S. (2007). The narrowing sex differential in life expectancy in high-income populations: effects of differences in the age pattern of mortality. Population studies, 61(2), 141-159. DOI: https://doi.org/10.1080/00324720701331433

Hinote B.P., Webber G.R. (2012). Drinking toward manhood: masculinity and alcohol in the former USSR. Men and Masculinities, 15(3), 292-310. DOI: https://doi.org/10.1177/1097184X12448466

Holmila M., Raitasalo K. (2005). Gender differences in drinking: why do they still exist? Addiction, 100(12), 1763-1769. DOI: https://doi.org/10.1111/j.13600443.2005.01249.x

Janssen F. (2020). Changing contribution of smoking to the sex differences in life expectancy in Europe, 1950-2014. European Journal of Epidemiology, 35, 835-841. DOI: https://doi.org/10.1007/s10654-020-00602-x

Kaplan R.M., Erickson P. (2000). Gender differences in quality-adjusted survival using a healthutilities index. American Journal of Preventive Medicine, 18, 77-82. DOI: https://doi.org/10.1016/s0749-3797(99)00101-4.

Kaufman M. (1994). Men, feminism, and men's contradictory experiences of power. In: H. Brod, M. Kaufman (Eds.), Theorising Masculinities (pp. 142-163). Sage Publications, Thousand Oaks, CA.

Koop C.E. (1996). Foreword. In: S.H. Woolf, S. Jonas, R.S. Lawrence (Eds.), Health Promotion and Disease Prevention in Clinical Practice (pp. 7-9). Williams and Wilkins, Baltimore, MD.

Korotayev A., Khaltourina D., Meshcherina K., Zamiatnina E. (2018). Distilled Spirits Overconsumption as the Most Important Factor of Excessive Adult Male Mortality in Europe. Alcohol and Alcoholism, 53(6), 742-752. DOI: https://doi.org/10.1093/alcalc/agy054

Luy M., Wegner-Siegmundt C. (2013). The impact of smoking and other non-biological factors on sex differences in life expectancy in Europe. Vienna Inst. of Demography. European Demographic Research Papers No 1/2013. URL: https://www.oeaw.ac.at/fileadmin/subsites/Institute/VID/PDF/Publications/EDRP/edrp_2013 _01.pdf

Mäkelä P., Gmel G., Grittner U., Kuendig H., Kuntsche S., Bloomfield K., Room R. (2006). Drinking patterns and their gender differences in Europe. Alcohol and Alcoholism, 41(suppl_1), i8-i18. DOI: https://doi.org/10.1093/alcalc/ag1071.

Marais G. A., Gaillard J. M., Vieira C., Plotton I., Sanlaville D., Gueyffier F., Lemaitre J. F. (2018). Sex gap in aging and longevity: can sex chromosomes play a role? Biology of sex differences, 9(1), 33. DOI: https://doi.org/10.1186/s13293-018-0181-y

McCartney G., Mahmood L., Leyland A. H., Batty G. D., Hunt K. (2011). Contribution of smoking-related and alcohol-related deaths to the gender gap in mortality: evidence from 30 European countries. Tobacco control, 20(2), 166-168. DOI: https://doi.org/10.1136/tc.2010.037929

Mehta P.H., Josephs R.A. (2011). Social endocrinology: Hormones and social motivation. In D. Dunning (Ed.), The handbook of social motivation (pp. 171-189). New York, NY: Psychology Press. 
Morris J.N. (1955). Uses of epidemiology. British Medical Journal, 2(4936), 395-401. DOI: https://doi.org/10.1136/bmj.2.4936.395.

Nathanson C. A. (1975). Illness and the feminine role: a theoretical review. Social Science \& Medicine, 9(2), 57-62. DOI: https://doi.org/10.1016/0037-7856(75)90094-3

National Center for Health Statistics (2003). Health United States. Hyattsville, MD: U.S. Public Health Service. URL: https://www.cdc.gov/nchs/data/hus/hus03.pdf

Oksuzyan A., Brønnum-Hansen H., Jeune B. (2010). Gender gap in health expectancy. European Journal of Ageing, 7, 213-218. DOI: https://doi.org/10.1007/s10433-010-0170-4

Philips S.P. (2006). Risky business: Explaining the gender gap in longevity. The Journal of Men's Health \& Gender, 3, 43-46. DOI: https://doi.org/10.1016/j.jmhg.2005.08.004

Pyke K.D. (1996). Class-based masculinities: the interdependence of gender, class and interpersonal power. Gender and Society, 10, 527-549. DOI: https://doi.org/10.1177/089124396010005003

Retherford R. D. (1972). Tobacco smoking and the sex mortality differential. Demography, 9, 203-216. DOI: https://doi.org/10.2307/2060633.

Rieker P.P., Bird C.E. (2005). Rethinking gender differences in health: why we need to integrate social and biological perspectives. The Journals of Gerontology Series B: Psychological Sciences and Social Sciences, 60(2), S40-S47. DOI: https://doi.org/10.1093/geronb/60.special_issue_2.s40

Rigby J.E., Dorling D. (2007). Mortality in relation to sex in the affluent world. Journal of Epidemiology \& Community Health, 61(2), 159-164. DOI: https://doi.org/10.1136/jech.2006.047381

Ristvedt S.L., Josephs R.A., Liening S.H. (2012). Endogenous testosterone levels are associated with assessments of unfavourable health information. Psychology \& Health, 27, 507-514. DOI: https://doi.org/10.1080/08870446.2012.657639

Rogers R.G., Everett B.G., Saint Onge J.M., Krueger P.M. (2010). Social, behavioral, and biological factors, and sex differences in mortality. Demography, 47, 555-578. DOI: https://doi.org/10.1353/dem.0.0119

Sampathkumar N.K., Bravo J.I., Chen Y., Danthi P.S., Donahue E.K., Lai R.W., Lu R., Randall L.T., Vinson N., Benayoun B.A. (2020). Widespread sex dimorphism in aging and agerelated diseases. Human genetics, 139(3), 1-24. DOI: https://doi.org/10.1007/s00439-01902082-w

Seifarth J.E., McGowan C.L., Milne K.J. (2012). Sex and life expectancy. Gender medicine, 9(6), 390-401. DOI: https://doi.org/10.1016/j.genm.2012.10.001.

Stanton S.J., Liening S.H., Schultheiss O.C. (2011). Testosterone is positively associated with risk taking in the Iowa Gambling Task. Hormones \& Behaviour, 59, 252-256. DOI: https://doi.org/10.1016/j.yhbeh.2010.12.003

Thorslund M., Wastesson J.W., Agahi N., Lagergren M., Parker M.G. (2013). The rise and fall of women's advantage: a comparison of national trends in life expectancy at age 65 years. European journal of ageing, 10(4), 271-277. DOI: https://doi.org/10.1007/s10433013-0274-8

Trias-Llimós S., Janssen F. (2018). Alcohol and gender gaps in life expectancy in eight Central and Eastern European countries. The European Journal of Public Health, 28(4), 687-692. DOI: https://doi.org/10.1093/eurpub/cky057 
Valkonen T., van Poppel F. (1997). The contribution of smoking to sex differences in life expectancy. Four nordic countries and the Netherlands 1970-1989. European Journal of Public Health, 7, 302-310. DOI: https://doi.org/10.1093/eurpub/7.3.302

van Wingen G.A., Ossewaarde L., Bäckström T., Hermans E.J., Fernández G. (2011). Gonadal hormone regulation of the emotion circuitry in humans. Neuroscience, 191, 38-45. DOI: https://doi.org/10.1016/j.neuroscience.2011.04.042

Verbrugge L.M., Wingard D.L. (1987). Sex differentials in health and mortality. Women \& health, 12(2), 103-145. DOI: https://doi.org/10.1300/J013v12n02_07

Waldron I. (1985). What do we know about causes of sex differences in mortality? A review of the literature. Population Bulletin of the United Nations, 18, 59-76.

Waldron I. (1986). The contribution of smoking to sex differences in mortality. Public Health Reports, 101, 163-173.

Wilsnack R.W., Vogeltanz N.D., Wilsnack S.C., Harris T.R. (2000). Gender differences in alcohol consumption and adverse drinking consequences: cross-cultural patterns. Addiction, 95(2), 251-265. DOI: https://doi.org/10.1046/j.1360-0443.2000.95225112.x

Wilsnack R.W., Wilsnack S.C., Kristjanson A.F., Vogeltanz-Holm N.D., Gmel G. (2009). Gender and alcohol consumption: patterns from the multinational GENACIS project. Addiction, 104(9), 1487-1500. DOI: https://doi.org/10.1111/j.13600443.2009.02696.x 


\title{
THE GENDER GAP IN LIFE EXPECTANCY: A REVIEW OF GENETIC, SOCIAL, AND VALUE FACTORS
}

\author{
JULIA ZINKINA, ANDREY KOROTAYEV
}

\begin{abstract}
A female advantage in life expectancy is found all over the world, despite differences in living conditions, women's status and other factors. However, the female morbidity rate is higher. This paradox is based on both biological and social factors. Among biological factors can be noted hormonal differences (the predominance of estrogens compared to androgens) and karyotypic differences - for example, progressive distortion of X-chromosome inactivation, telomere depletion, mitochondrial inheritance, etc. Health behavior makes a significant contribution: men are less likely to see a doctor, less likely to control cholesterol and blood pressure, tend to have a less healthy diet, and are more likely to engage in risky behaviors. A special contribution is made by smoking (in Russia it causes up to a 5-year gap in life expectancy between men and women, and in Europe is still the most important cause of gender differences in mortality) and alcohol consumption (men drink more often than women, and male drinkers consume alcohol more often, in larger quantities and with a higher risk of adverse effects than female drinkers; in some countries, this factor explains up to $30 \%$ of the gender gap in life expectancy). Value explanations for the factors of gender differences in life expectancy are not limited to data from cross-national value surveys. It seems appropriate to include in this category explanations associated with the dominant notions of masculinity.
\end{abstract}

Key words: gender gap in life expectancy, biological factors of the gender gap in life expectancy, social factors of the gender gap in life expectancy, smoking as a factor of the gender gap in life expectancy, alcohol consumption as a factor of the gender gap in life expectancy.

Julia Zinkina (juliazin@list.ru), Russian Presidential ACADEMy of NATIONAL ECONOMY AND PubliC ADMINISTRATION, RUSSIA.

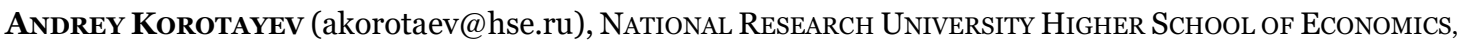
Russian PRESIDENTIAL ACADEMy OF NATIONAL ECONOMY AND PUblic AdMINISTRATION, Russia.

THE ARTICLE HAS BEEN PREPARED WITHIN THE FRAMEWORK OF THE RESEARCH WORK OF THE STATE TASK RANEPA.

DATE RECEIVED : DECEMBER 2020.

\section{REFERENCES}

Andreev E.M. (2001). Smertnost' muzhchin v Rossii. Voprosy statistiki, 7, 27-33. (In Russ.).

Andreev E.M., Shkolnikov V.M., McKee M. (2002). Prodolzhitel'nost' zdorovoj zhizni. Voprosy statistiki, 11, 16-21. (In Russ.).

Arias E. (2007). United States Life Tables, 2004. National Vital Statistics Reports, 54(14), 1-40.

Cullen M.R., Baiocchi M., Eggleston K., Loftus P., Fuchs V. (2015). The weaker sex? Vulnerable men, resilient women, and variations in sex differences in mortality since 1900 (No. w21114). National Bureau of Economic Research.

Austad S.N. (2006). Why women live longer than men: sex differences in longevity. Gender medicine, 3(2), 79-92. DOI: https://doi.org/10.1016/s1550-8579(06)80198-1

Brown J.V., Panova L.V., Rusinova N.L. (2007). Gendernye razlichija v zdorov'e. Sociologicheskie issledovanija, 6, 114-122. (In Russ.). 
Courtenay W.H. (2000). Behavioral factors associated with disease, injury, and death among men: Evidence and implications for prevention. The Journal of Men's Studies, 9(1), 81-142. DOI: https://doi.org/10.3149/jms.0901.81

Emslie C., Hunt K. (2008). The weaker sex? Exploring lay understandings of gender differences in life expectancy: a qualitative study. Social Science \& Medicine, 67(5), 808-816. DOI: https://doi.org/10.1016/j.socscimed.2008.05.009.

Erol A., Karpyak V.M. (2015). Sex and gender-related differences in alcohol use and its consequences: Contemporary knowledge and future research considerations. Drug and alcohol dependence, 156, 1-13. DOI: https://doi.org/10.1016/j.drugalcdep.2015.08.023

Gardner M., Bann D., Wiley L., Cooper R., Hardy R., Nitsch D., Bekaert S. (2014). Gender and telomere length: systematic review and meta-analysis. Experimental gerontology, 51, 15-27. DOI: https://doi.org/10.1016/j.exger.2013.12.004

Glei D.A., Horiuchi S. (2007). The narrowing sex differential in life expectancy in high-income populations: effects of differences in the age pattern of mortality. Population studies, 61(2), 141-159. DOI: https://doi.org/10.1080/00324720701331433

Hinote B.P., Webber G.R. (2012). Drinking toward manhood: masculinity and alcohol in the former USSR. Men and Masculinities, 15(3), 292-310. DOI: https://doi.org/10.1177/1097184X12448466

Holmila M., Raitasalo K. (2005). Gender differences in drinking: why do they still exist? Addiction, 100(12), 1763-1769. DOI: https://doi.org/10.1111/j.13600443.2005.01249.x

Janssen F. (2020). Changing contribution of smoking to the sex differences in life expectancy in Europe, 1950-2014. European Journal of Epidemiology, 35, 835-841. DOI: https://doi.org/10.1007/s10654-020-00602-x

Kaplan R.M., Erickson P. (2000). Gender differences in quality-adjusted survival using a healthutilities index. American Journal of Preventive Medicine, 18, 77-82. DOI: https://doi.org/10.1016/s0749-3797(99)00101-4.

Kaufman M. (1994). Men, feminism, and men's contradictory experiences of power. In: H. Brod, M. Kaufman (Eds.), Theorising Masculinities (pp. 142-163). Sage Publications, Thousand Oaks, CA.

Khaltourina D.A., Korotayev A.V. (2013). Russkiy krest. Rossiyskiy demograficheskiy krizis: faktory, mekhanizmy, puti preodoleniya. Librokom/URSS, Moscow. (In Russ.).

Kharchenko V.I., Mikhailova R.Yu., Onishchenko P.I. (2003). Pokazateli prodolzhitel'nosti zhizni naselenija Rossii v sravnenii s drugimi stranami. Problemy prognozirovanija, 6, 119127. (In Russ.).

Khasanova R.R. (2019). Prodolzhitel'nost' zhizni: differenciacija po polu. Ekonomicheskoe razvitie Rossii, 26(5), 56-60. (In Russ).

Koop C.E. (1996). Foreword. In: S.H. Woolf, S. Jonas, R.S. Lawrence (Eds.), Health Promotion and Disease Prevention in Clinical Practice (pp. 7-9). Williams and Wilkins, Baltimore, MD.

Korotayev A., Khaltourina D., Meshcherina K., Zamiatnina E. (2018). Distilled Spirits Overconsumption as the Most Important Factor of Excessive Adult Male Mortality in Europe. Alcohol and Alcoholism, 53(6), 742-752. DOI: https://doi.org/10.1093/alcalc/agy054 
Kossova T.V., Kossova E.V., Sheluncova M.A. (2018). Analiz faktorov, opredeljajushhih razlichie v ozhidaemoj prodolzhitel'nosti muzhchin i zhenshhin v regionah Rossii. EKO, 4, 116-132. (In Russ.). DOI: http://dx.doi.org/10.30680/ECO0131-7652-2018-4-116-132

Kuznetsova P. (2019). Smoking as a factor of reduced life expectancy in Russia. Demographic Review, 6(3), 31-57. https://doi.org/10.17323/demreview.v6i3.9854

Luy M., Wegner-Siegmundt C. (2013). The impact of smoking and other non-biological factors on sex differences in life expectancy in Europe. Vienna Inst. of Demography. European Demographic Research Papers No 1/2013. URL:

https://www.oeaw.ac.at/fileadmin/subsites/Institute/VID/PDF/Publications/EDRP/edrp_2013 _01.pdf

Mäkelä P., Gmel G., Grittner U., Kuendig H., Kuntsche S., Bloomfield K., Room R. (2006). Drinking patterns and their gender differences in Europe. Alcohol and Alcoholism, 41(suppl_1), i8-i18. DOI: https://doi.org/10.1093/alcalc/ag1071.

Marais G.A., Gaillard J.M., Vieira C., Plotton I., Sanlaville D., Gueyffier F., Lemaitre J.F. (2018). Sex gap in aging and longevity: can sex chromosomes play a role? Biology of sex differences, 9(1), 33. DOI: https://doi.org/10.1186/s13293-018-0181-y

McCartney G., Mahmood L., Leyland A.H., Batty G.D., Hunt K. (2011). Contribution of smoking-related and alcohol-related deaths to the gender gap in mortality: evidence from 30 European countries. Tobacco control, 20(2), 166-168. DOI: https://doi.org/10.1136/tc.2010.037929

Mehta P.H., Josephs R.A. (2011). Social endocrinology: Hormones and social motivation. In D. Dunning (Ed.), The handbook of social motivation (pp. 171-189). New York, NY: Psychology Press.

Morris J.N. (1955). Uses of epidemiology. British Medical Journal, 2(4936), 395-401. DOI: https://doi.org/10.1136/bmj.2.4936.395.

Nathanson C.A. (1975). Illness and the feminine role: a theoretical review. Social Science \& Medicine, 9(2), 57-62. DOI: https://doi.org/10.1016/0037-7856(75)90094-3

National Center for Health Statistics (2003). Health United States. Hyattsville, MD: U.S. Public Health Service. URL: https://www.cdc.gov/nchs/data/hus/hus03.pdf

Oksuzyan A., Brønnum-Hansen H., Jeune B. (2010). Gender gap in health expectancy. European Journal of Ageing, 7, 213-218. DOI: https://doi.org/10.1007/s10433-010-0170-4

Philips S.P. (2006). Risky business: Explaining the gender gap in longevity. The Journal of Men's Health \& Gender, 3, 43-46. DOI: https://doi.org/10.1016/j.jmhg.2005.08.004

Pyke K.D. (1996). Class-based masculinities: the interdependence of gender, class and interpersonal power. Gender and Society, 10, 527-549. DOI: https://doi.org/10.1177/089124396010005003

Retherford R.D. (1972). Tobacco smoking and the sex mortality differential. Demography, 9, 203-216. DOI: https://doi.org/10.2307/2060633.

Rieker P.P., Bird C.E. (2005). Rethinking gender differences in health: why we need to integrate social and biological perspectives. The Journals of Gerontology Series B: Psychological Sciences and Social Sciences, 60(2), S40-S47. DOI: https://doi.org/10.1093/geronb/60.special_issue_2.s40 
Rigby J.E., Dorling D. (2007). Mortality in relation to sex in the affluent world. Journal of Epidemiology \& Community Health, 61(2), 159-164. DOI:

https://doi.org/10.1136/jech.2006.047381

Ristvedt S.L., Josephs R.A., Liening S.H. (2012). Endogenous testosterone levels are associated with assessments of unfavourable health information. Psychology \& Health, 27, 507-514. DOI: https://doi.org/10.1080/08870446.2012.657639

Rogers R.G., Everett B.G., Saint Onge J.M., Krueger P.M. (2010). Social, behavioral, and biological factors, and sex differences in mortality. Demography, 47, 555-578. DOI: https://doi.org/10.1353/dem.0.0119

Sampathkumar N.K., Bravo J.I., Chen Y., Danthi P.S., Donahue E.K., Lai R.W., Lu R., Randall L.T., Vinson N., Benayoun B.A. (2020). Widespread sex dimorphism in aging and agerelated diseases. Human genetics, 139(3), 1-24. DOI: https://doi.org/10.1007/s00439-01902082-w

Seifarth J.E., McGowan C.L., Milne K.J. (2012). Sex and life expectancy. Gender medicine, 9(6), 390-401. DOI: https://doi.org/10.1016/j.genm.2012.10.001.

Shkolnikov V., Andreev E., Maleva T. (Eds.) (2000). Neravenstvo i smertnost v Rossii [Inequality and mortality in Russia]. Moscow: Signal. (In Russ.).

Shkolnikov V.M., Andreev E.M., McKee M., Leon D.A. Rising life expectancy in Russia of the 2000s. Demographic Review, 1(2), 5-37. (In Russ)

https://doi.org/10.17323/demreview.v1i2.1815

Shkolnikov V., Mesle F., Vallin J. (1995). Ozhidaemaja prodolzhitel'nost' zhizni i smertnost' naselenija_Rossii v 1970 - 1993 godah: analiz i prognoz [Life expectancy and mortality of Russian population in 1970 - 1993: analysis and forecast]. Moscow: Fond «Zdorovje i okruzhaushchaya sreda». (In Russ.).

Stanton S.J., Liening S.H., Schultheiss O.C. (2011). Testosterone is positively associated with risk taking in the Iowa Gambling Task. Hormones \& Behaviour, 59, 252-256. DOI: https://doi.org/10.1016/j.yhbeh.2010.12.003

Thorslund M., Wastesson J. W., Agahi N., Lagergren M., Parker M. G. (2013). The rise and fall of women's advantage: a comparison of national trends in life expectancy at age 65 years. European journal of ageing, 10(4), 271-277. DOI: https://doi.org/10.1007/s10433013-0274-8

Trias-Llimós S., Janssen F. (2018). Alcohol and gender gaps in life expectancy in eight Central and Eastern European countries. The European Journal of Public Health, 28(4), 687-692. DOI: https://doi.org/10.1093/eurpub/cky057

Valkonen T., van Poppel F. (1997). The contribution of smoking to sex differences in life expectancy. Four nordic countries and the Netherlands 1970-1989. European Journal of Public Health, 7, 302-310. DOI: https://doi.org/10.1093/eurpub/7.3.302

van Wingen G.A., Ossewaarde L., Bäckström T., Hermans E.J., Fernández G. (2011). Gonadal hormone regulation of the emotion circuitry in humans. Neuroscience, 191, 38-45. DOI: https://doi.org/10.1016/j.neuroscience.2011.04.042

Verbrugge L.M., Wingard D.L. (1987). Sex differentials in health and mortality. Women \& health, 12(2), 103-145. DOI: https://doi.org/10.1300/J013v12n02_07

Waldron I. (1985). What do we know about causes of sex differences in mortality? A review of the literature. Population Bulletin of the United Nations, 18, 59-76. 
Waldron I. (1986). The contribution of smoking to sex differences in mortality. Public Health Reports, 101, 163-173.

Wilsnack R.W., Vogeltanz N.D., Wilsnack S.C., Harris T.R. (2000). Gender differences in alcohol consumption and adverse drinking consequences: cross-cultural patterns. Addiction, 95(2), 251-265. DOI: https://doi.org/10.1046/j.1360-0443.2000.95225112.x

Wilsnack R.W., Wilsnack S.C., Kristjanson A.F., Vogeltanz-Holm N.D., Gmel G. (2009). Gender and alcohol consumption: patterns from the multinational GENACIS project. Addiction, 104(9), 1487-1500. DOI: https://doi.org/10.1111/j.13600443.2009.02696.x 